\title{
Research on Flexible Reliability Evaluation of Distribution Network System with Distributed Energy Access
}

\author{
Gu Zhihong*1, Wen Wei ${ }^{1}$, Liu Zhuo ${ }^{1}$ \\ ${ }^{1}$ State Grid Shanxi Economic Research Institute, 030002, China
}

\begin{abstract}
Access to distributed energy has brought a greater impact on the system. By analyzing the flexibility of the distribution network itself, considering the evaluation indicators of the three dimensions of effect, degree and service, this paper designs a flexible reliability evaluation index system for the distribution network, using principal component analysis and multi-level fuzzy comprehensive evaluation thoughts, a flexible reliability model was established, and calculation examples were analyzed, which is helpful to provide guidance for the development of regional distribution networks.
\end{abstract}

\section{Introduction}

With the continuous increase of wind power and photovoltaic installed capacity, it has brought a greater impact to the system. According to data from the National Energy Administration, the grid-connected wind power and photovoltaic installed capacity reached $210.05 \mathrm{GW}$ and $204.68 \mathrm{GW}$ respectively at the end of 2019. Wind power, photovoltaics and other new energy sources show obvious instability and intermittent, and access to the distribution network system directly affects the power quality [1]. Moreover, as an important characterization of the distribution network, flexibility and reliability have not yet formed a unified definition. Therefore, it is urgent to analyze the current development status of large-scale access to distributed energy in the current distribution network system, and further quantify and evaluate the flexible reliability of the distribution network system.

At present, many scholars have carried out research on the reliability of the distribution network system. Luo et al [2] designed a reliability evaluation index system based on the redundant phenomenon of traditional distribution network operation reliability evaluation indicators, starting from the actual experience of users and the construction of the distribution network in Chengdu. This index system adopted cluster analysis and improved grey relational degree method for case analysis. Xiao et al [3] comprehensively considered the five dimensions of safety, economy, reliability, adaptability and quality, and used principal component analysis to analyze the internal relationship between the indicators of the distribution network, and calculated the weight of each indicator. Based on actual needs, Lei et al [4] proposed a county-level distribution network planning evaluation index system and scoring method combining the characteristics of the county-level distribution network. Zhang et al [5] proposed an evaluation index system for the construction effect of distribution automation based on SMART criteria. Based on the quantitative analysis of reliability statistical indicators, $\mathrm{Wu}$ et al [6] proposed the implementation degree index of reliability improvement measures. Chen et al [7] considered the four dimensions of economy, service quality, safety, and environmental benefits, and conducted a comprehensive evaluation of a distribution network with distributed power sources. Although many of the above studies have conducted in-depth discussions on the reliability of the distribution network, they failed to comprehensively consider the flexibility of the distribution network, which led to the deviation of the evaluation results from the actual situation.

Therefore, on the basis of analyzing the uncertain characteristics brought by wind power and photovoltaic access to the distribution network system, this paper considers the evaluation indicators of the three dimensions of effect, degree and service, using principal component analysis and multi-level fuzzy comprehensive method establishes a flexible reliability evaluation model of the distribution network system with distributed energy access, and selects a regional distribution network of $\mathrm{A}, \mathrm{B}$, and $\mathrm{C}$ for empirical analysis, which helps to enhance the flexibility and reliability of the regional distribution network.

* Corresponding author: 381593857@qq.com 


\section{Design a flexible reliability evaluation index system for distribution network}

In view of the flexible characteristics of the distribution network, relevant research literature and research information are collected, and the evaluation indicators that can characterize its reliability can be roughly divided into effect indicators, degree indicators and service indicators, as shown in Figure 1.

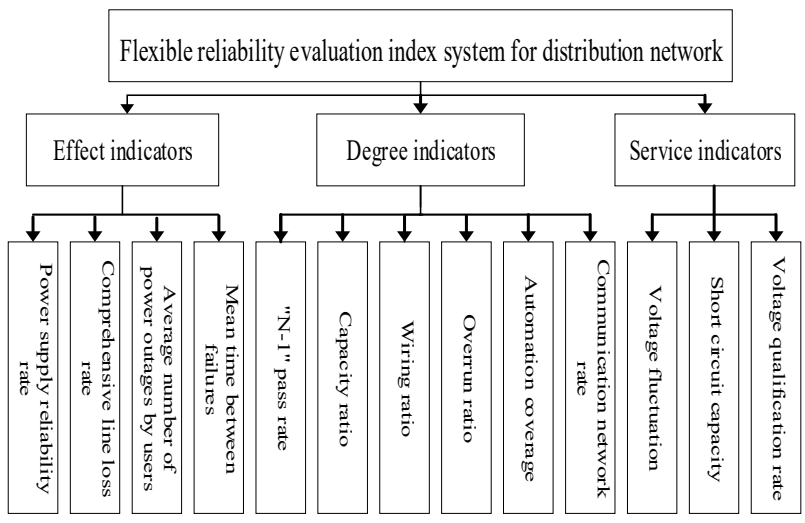

Fig 1 Flexible reliability evaluation index system for distribution network

(1) Effect indicators

The effect index better characterizes the quantitative results of the flexible reliability of the distribution network, mainly involving four evaluation indexes such as power supply reliability rate, comprehensive line loss rate, average number of user outages and average outage time [8]. Among them, the power supply reliability rate is an important quantitative indicator of power supply reliability, which represents the continuous power supply capacity measurement, and the specific calculation method is:

$$
K e=(T y / T s) * 100 \%
$$

In the formula, ${ }^{T y}$ represents the sum of the actual power supply time during the statistical period; $T S$ represents the total power supply time during the statistical period.

The comprehensive line loss rate is an important indicator to measure the management level of the power supply unit. $10 \mathrm{kV}$ is $10 \%$, and $380 / 220 \mathrm{~V}$ is $12 \%$; the average number of power outages of users needs to count the number of power outages and the total number of power users in the region, which directly reflects the reliability index of the power supply system to users. The mean time between failures needs to count the average power failure hours of each user.

\section{(2) Degree indicators}

The degree index directly characterizes the results of the flexible reliability of the distribution network, mainly involving six evaluations such as "N-1" pass rate, capacity ratio, wiring rate, overrun rate, automation coverage rate and communication network rate index. Among them, the "N-1" pass rate is an important criterion for judging its safety in the distribution system, which is generally divided into main transformers and lines. When any one of the lines is disconnected and causes the other lines of the system to be overloaded or the system is disconnected, it means that the network does not meet the N-1 test. When the above situation occurs, a certain grid planning method must be applied to expand the network until the N-1 inspection is passed.

The capacity-to-load ratio is the ratio of the available transformation capacity of a certain voltage level $(10 \mathrm{kV}$, $110 \mathrm{kV}, 220 \mathrm{kV}$ ) grid to the corresponding maximum load on the basis of power supply reliability. When the capacity-load ratio of the distribution network is too large and the available capacity is too much, it reflects the phenomena of too much advance in the construction of transformation and distribution projects and premature investment in construction costs.

The wiring ratio generally refers to the singleradiation wiring ratio. The specific calculation method is that the ratio of single-radiation wiring lines $(\%)=$ the number of single-radiation wiring lines/total number of lines $\times 100 \%$.

The overrun ratio generally means that the ratio of resistance to reactance in the network is larger, that is, the resistance is large and the reactance is relatively small.

Automation coverage and communication networking generally refer to the realization of monitoring and control of the distribution system based on the primary grid and equipment, with the distribution network automation system as the core.

(3) Service indicators

Service indicators characterize the user experience of the flexible reliability of the distribution network, mainly involving three evaluation indicators such as voltage fluctuation, short-circuit capacity and voltage qualification rate. Among them, voltage fluctuation refers to the rapid change of the effective value of the grid voltage (root mean square value). The short-circuit capacity is used to calculate the relationship between reactive power impulse and voltage fluctuation. When a short-circuit fault occurs in the power system, it is necessary to quickly cut off the faulty part so that the rest can continue to operate. The voltage qualification rate refers to the percentage of the total time that the voltage at the monitoring point is within the qualified range and the total monthly voltage monitoring time within one month during the operation of the power grid. In general, the reliability of power supply in urban areas and rural areas is not less than $99.9 \%$ and $99.58 \%$, respectively, and the voltage qualification rate of residential customers is not less than $96 \%$ and $93.89 \%$.

\section{Flexible reliability evaluation model based on principal component analysis and fuzzy integrated distribution network}

\subsection{Calculate the weight of evaluation index}

Analyze the massive index data of the flexible reliability of the distribution network to make the weights more reliable and avoid subjective judgments. In this paper, 
according to the principal component analysis method, the variance and cumulative contribution rate table and factor loading matrix table of each flexible reliability index are obtained, so as to calculate the weight of each index in the effect category, degree category and service category. The specific calculation steps are as follows [9]:

(1) Divide the load numbers in the factor load matrix table by the square roots of the corresponding principal component characteristic roots in the variance and cumulative contribution rate tables to obtain the coefficients of each index in the linear combination of principal components.

(2) Multiply the coefficient of each indicator calculated in step (1) by the variance contribution rate of the corresponding principal component, and divide by the sum of the variance contribution rates to obtain the weight of each indicator.

(3) Normalize and remove the dimension of the income weight, namely:

The reverse evaluation index is processed forward, namely:

$$
y_{i j}=\max \left\{x_{i j}\right\}-x_{i j}
$$

Proper index processing, namely:

$$
y_{i j}=\max \left|x_{i j}-k\right|-\left|x_{i j}-k\right|
$$

In the formula, $x_{i j}$ and $y_{i j}$ represent the index values before and after the evaluation index unification process, and $k$ represents the appropriate value of the appropriate index.

\subsection{Calculate the evaluation result vector}

In this paper, the multi-level fuzzy comprehensive evaluation method is used to comprehensively evaluate the subordination status of the flexible reliability of the distribution network from the three dimensions of the effect category, the degree category and the service category, so as to realize the evaluation of the overall status of the distribution network. The calculation steps as follows:

(1) Set up a set of index factors. Assuming that the object to be evaluated is an object set $P$, determine each index related to $P$ to establish an evaluation factor set, namely an index set $U=\left(u_{1}, u_{2}, \ldots, u_{m}\right)$, and select a set of possible evaluation results to establish an evaluation level set $V=\left(v_{1}, v_{2}, \ldots, v_{n}\right)$.

(2) Establish the membership degree relationship matrix $R$, and evaluate the membership degree of each index in $U$ according to each level in the evaluation level set $V$, namely:

$$
R=\left(\begin{array}{c}
R_{1} \\
R_{2} \\
\vdots \\
R_{m}
\end{array}\right)=\left(\begin{array}{cccc}
r_{11} & r_{12} & \ldots & r_{1 n} \\
r_{21} & r_{22} & \ldots & r_{2 n} \\
\vdots & \vdots & \ddots & \vdots \\
r_{m 1} & r_{m 2} & \ldots & r_{m n}
\end{array}\right)
$$

In the formula, $r_{i j}$ represents the degree of membership of ${ }^{u_{i}}$ to the evaluation level in the set $V$ and its corresponding value $v_{j}$, and $r_{i 1}+r_{i 2}+\ldots+r_{i n}=1$.

(3) Establish a weight vector $W$, apply the principal component analysis method to calculate $u_{i}$ corresponding weight $w_{i}$ to reflect the importance of each sub-index $u_{i}$ to $P$, and the weight vector is $W=\left(w_{1}, w_{2}, \ldots, w_{m}\right)$.

(4) Establish the evaluation result item $B$, based on the membership degree relationship matrix $R$ and the weight vector $W$, the composite operation obtains the evaluation result vector $B$, namely:

$$
B=W R=\left(w_{1}, w_{2}, \ldots, w_{m}\right)\left(\begin{array}{cccc}
r_{11} & r_{12} & \ldots & r_{1 n} \\
r_{21} & r_{22} & \ldots & r_{2 n} \\
\vdots & \vdots & \ddots & \vdots \\
r_{m 1} & r_{m 2} & \ldots & r_{m n}
\end{array}\right)
$$

\subsection{Flexible reliability index evaluation}

In summary, the principal component analysis method and multi-level fuzzy comprehensive evaluation method used in this paper are used to evaluate the flexible reliability index of the distribution network. According to the principle of maximum membership degree, the evaluation level corresponding to the maximum value in $B$ is taken as the evaluation result.

\subsection{Specific calculation process}

The flow chart of flexible reliability assessment of distribution network based on principal component analysis and fuzzy comprehensive evaluation is shown in Figure 2 .

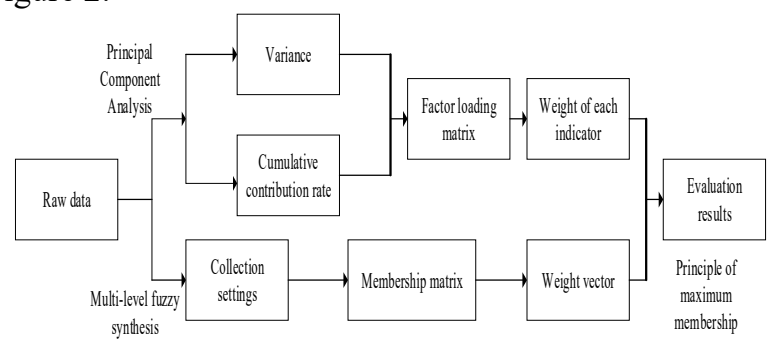

Fig 2 Flow chart of flexible reliability assessment of distribution network

\section{Case analysis}

In order to verify the effectiveness of the proposed flexible reliability evaluation model for the distribution network, a regional distribution network is selected for analysis. 


\subsection{Determine the weight of flexible reliability index}

Principal component analysis is carried out with degree indicators, and the corresponding variance and cumulative contribution rate are shown in Table 1.

Table 1 Variance and cumulative contribution rate of degree indicators

\begin{tabular}{|c|c|c|c|}
\hline \multirow{2}{*}{ NUM } & \multicolumn{3}{|c|}{ Initial eigenvalue } \\
\hline & Total & Percentage of variance & Grand total \\
\hline 1 & 3.75 & 33.68 & 24.32 \\
\hline 2 & 2.69 & 20.42 & 46.78 \\
\hline 3 & 2.50 & 16.70 & 62.36 \\
\hline 4 & 1.73 & 12.27 & 73.62 \\
\hline 5 & 1.70 & 12.36 & 89.31 \\
\hline 6 & 0.61 & 4.54 & 100.0 \\
\hline \multirow{2}{*}{ NUM } & \multicolumn{3}{|c|}{ Extract the sum of squares and load } \\
\hline & Total & Percentage of variance & Grand total \\
\hline 1 & 3.75 & 33.68 & 24.32 \\
\hline 2 & 2.69 & 20.42 & 46.78 \\
\hline 3 & 2.50 & 16.70 & 62.36 \\
\hline \multicolumn{4}{|l|}{4} \\
\hline \multicolumn{4}{|l|}{5} \\
\hline \multicolumn{4}{|l|}{6} \\
\hline \multirow{2}{*}{ NUM } & \multicolumn{3}{|c|}{ Rotate the sum of squares loading } \\
\hline & Total & Percentage of variance & Grand total \\
\hline 1 & 2.22 & 29.72 & 26.73 \\
\hline 2 & 1.16 & 18.12 & 48.92 \\
\hline 3 & 1.12 & 14.51 & 62.36 \\
\hline 4 & & & \\
\hline 5 & & & \\
\hline 6 & & & \\
\hline
\end{tabular}

It can be seen from the above results that the principal component analysis method proposes that the cumulative variance contribution rate of the three principal components accounts for $62.36 \%$ of the total variance, which more comprehensively reflects the relevant information of the degree indicators, so that the principal component factor loading matrix of the degree index can be obtained. The factor loading matrix is shown in Table 2.

Table 2 Principal component factor loading matrix of degree indicators

\begin{tabular}{ccccc}
\hline \multirow{2}{*}{ Num } & \multirow{2}{*}{$\begin{array}{c}\text { Degree } \\
\text { indicators }\end{array}$} & \multicolumn{3}{c}{ Main ingredient } \\
\cline { 3 - 5 } & "N-1" pass rate & 0.432 & 0.326 & -0.249 \\
\hline 1 & Capacity ratio & 0.256 & -0137 & -0.246 \\
\hline 2 & Wiring ratio & -0.152 & 0.377 & 0.411 \\
\hline 3 & Overrun ratio & 0.213 & -0.331 & 0.322 \\
\hline 4 & $\begin{array}{c}\text { Automation } \\
\text { coverage }\end{array}$ & 0.344 & 0.172 & -0.183 \\
\hline 5 & $\begin{array}{c}\text { Communication } \\
\text { network rate }\end{array}$ & 0.174 & 0.259 & -0.223 \\
\hline 6
\end{tabular}

According to the variance, cumulative contribution rate and principal component factor loading matrix of the degree indicators, the principal component analysis method can be used to obtain the weights of the corresponding indicators of the effect, degree and service categories, as shown in Table 3.

Table 3 The weight of each effect indicator

\begin{tabular}{lrr}
\hline Number & Effect indicators & $\begin{array}{c}\text { Index } \\
\text { weight }\end{array}$ \\
\hline
\end{tabular}

\begin{tabular}{ccc}
\hline 1 & Power supply reliability rate & 0.436 \\
\hline 2 & Comprehensive line loss rate & 0.188 \\
\hline 3 & $\begin{array}{c}\text { Average number of power } \\
\text { outages by users }\end{array}$ & 0.204 \\
\hline 4 & Mean time between failures & 0.172 \\
\hline \multicolumn{3}{c}{ Table 4 The weight of each degree indicator } \\
\hline Number & Degree indicators & $\begin{array}{c}\text { Index } \\
\text { weight }\end{array}$ \\
\hline 1 & "N-1" pass rate & 0.256 \\
\hline 2 & Capacity ratio & 0.118 \\
\hline 3 & Wiring ratio & 0.177 \\
\hline 4 & Overrun ratio & 0.122 \\
\hline 5 & Automation coverage & 0.215 \\
\hline 6 & Communication network rate & 0.112 \\
\hline
\end{tabular}

Table 5 The weight of each service indicator

\begin{tabular}{ccc}
\hline Number & Service indicators & Index weight \\
\hline 1 & Voltage fluctuation & 0.440 \\
\hline 2 & $\begin{array}{c}\text { Short circuit } \\
\text { capacity }\end{array}$ & 0.319 \\
\hline 3 & $\begin{array}{c}\text { Voltage } \\
\text { qualification rate }\end{array}$ & 0.241 \\
\hline
\end{tabular}

\subsection{Determining flexible reliability index benefit evaluation}

Combined with the flexible reliability evaluation level set in the region, it is mainly divided into: \{good, better, general $\}=\{(100,85] ;[85,75] ;(75,60]\}$, so that the weight vector of each indicator of the effect category, degree category and service category can be obtained, as shown below:

$$
\begin{gathered}
W_{1}=(0.436,0.188,0.204,0.172), R_{1}=\left(\begin{array}{lll}
0.65 & 0.24 & 0.11 \\
0.43 & 0.42 & 0.15 \\
0.56 & 0.24 & 0.18 \\
0.47 & 0.37 & 0.16
\end{array}\right) \\
W_{2}=(0.256,0.118,0.177,0.122,0.215,0.112), R_{2}=\left(\begin{array}{lll}
0.72 & 0.16 & 0.12 \\
0.57 & 0.26 & 0.17 \\
0.55 & 0.29 & 0.16 \\
0.42 & 0.36 & 0.22 \\
0.53 & 0.39 & 0.08 \\
0.44 & 0.37 & 0.19
\end{array}\right) \\
W_{3}=(0.440,0.319,0.241), R_{3}=\left(\begin{array}{lll}
0.62 & 0.26 & 0.16 \\
0.55 & 0.27 & 0.18 \\
0.58 & 0.31 & 0.11
\end{array}\right)
\end{gathered}
$$

Get, namely:

$$
\begin{gathered}
B_{1}=(0.560,0.300,0.140), B_{2}=(0.563,0.292,0.144), \\
B_{3}=(0.588,0.275,0.154)
\end{gathered}
$$

It can be seen that the final flexible reliability index of the distribution network is at a "better" level, which further illustrates the strong flexibility and reliability of the distribution network in this region. According to the evaluation indicators obtained above, the power supply reliability rate in this area is relatively high and 
relatively stable. At the same time, from the perspective of service indicators, when the grid is further expanded, it is necessary to pay attention to the voltage stability and improve the stability and safety of power supply as much as possible.

\section{Conclusion}

Based on the flexibility of the distribution network system itself and taking into account the distributed energy access operation system, a flexible reliability evaluation method for the distribution network is proposed, which combines principal component analysis and multi-level fuzzy comprehensive thinking. The specific results are as follows:

(1) Based on the flexibility of the distribution network, considering 13 evaluation indicators from the three dimensions of effect, degree, and service, a flexible reliability index system for the distribution network is designed;

(2) Based on principal component analysis and multilevel fuzzy comprehensive evaluation ideas, a flexible reliability evaluation model for the distribution network is established.

(3) The results of the calculation examples verify that the flexible reliability level of the distribution network in this area is in a "better" state.

\section{Acknowledgement}

Project Supported by Research on device asset performance evaluation model and configuration optimization technology of Shanxi distribution network under Energy Internet (SGTYHT/18-JS-206).

\section{Reference}

1. Li Guixin, Ge Leijiao, et al. Research on the planning of $\mathrm{AC} / \mathrm{DC}$ hybrid distribution network considering the comprehensive utilizat[J]. Renewable Energy Resources, ,2020,38(01):98-103.

2. LUO Ning, CHEN Ludong, et al. Reliability evaluation of distribution network operation based on cluster analysis and improved grey[J]. Engineering Journal of Wuhan University, 2020,53(07):636-642.

3. XIAO Yingtao, WANG Huaquan, et al. Evaluation of Distribution Network Status Based on Principal Component Analysis and Correspondence Analysis [J]. Southern Energy Construction, 2019,6(03):105112.

4. LEI Qing-sheng, YAN Jing, et al. Differentiated evaluation system and grading method in county distribution network planning[J]. Journal of Electric Power Science and Technology, 2017,32(04):125131.

5. ZHANG Hongbin, WU Zhili, et al. Research on Power Distribution Automation Construction Effects
Evaluation System Based on SMART Criteria[J]. Power System Technology ,2016, 40(07):2192-2198.

6. WU Han, CHEN Bin, et al. Quantitative evaluation and optimized selection of power supply reliability enhancement measures[J]. Electric Power Automation Equipment ,2015,35(05):126-130.

7. CHEN Chiye, WEN Yafeng, et al. Comprehensive Evaluation Method of Distribution Network Including Various Types of Distributed Generation $[\mathrm{J}]$. Electric Power Construction, 2015,36(01):128-135.

8. SUN Yuxiao, ZHU Junpeng, et al. Reliability Evaluation Based on Dynamic Island MILP Model of Active Distribution Network[J]. Electric Power Construction, 2019,40(05):90-97.

9. Cherdkeattikul Supitchaya, Morisawa Yusuke, et al. Surface Structural Transformation of Pre-carbonized Solid Biomass from Japanese Cedar via ATR-FTIR and PCA.[J]. Analytical sciences : the international journal of the Japan Society for Analytical Chemistry, 2020, 36(6). 